\title{
POLYARTHRITIS FOLLOWING PEMBROLIZUMAB: A NEW PIECE IN THE PUZZLE OF RHEUMATOLOGISTS: A CASE REPORT
}

\author{
Jucier Gonçalves Júnior ${ }^{1, \star}$, Heitor Furlan Giordano ${ }^{1}$, Murillo Dório ${ }^{1}$ \\ 1.Universidade de São Paulo, São Paulo (SP), Brazil. \\ *Corresponding author: juciergjunior@hotmail.com
}

\section{BACKGROUND}

Checkpoint inhibitors (CPI) are drugs used for immunotherapy of advanced-stage neoplasms, such as melanoma, lung and kidney cancers, and several immune mediated manifestations have been reported as related adverse events. Among these medications, pembrolizumab interacts with the programmed cell death receptor (PD-1) and inhibits T cell apoptosis induced by tumor cells, leading to activation of $\mathrm{T}$ cells. Thus, the objective was to report a case of polyarthritis induced by pembrolizumab.

\section{CASE REPORT}

Female patient, 68 years old, white, with previous treatment of diffuse large B-cell lymphoma (stage IIIA) 4 years ago and current thymic carcinoma with surgical resection and radiotherapy for 3 years. One year ago, recurrence of thymic carcinoma was evidenced by pleural hyperuptake on PET-CT and pembrolizumab was started every 3 weeks. Ten days after the first infusion, the patient developed symmetrical, additive polyarthritis, starting in the knees and ankles bilaterally, progressing to the metacarpophalangeal and proximal interphalangeal joints, associated with morning stiffness (more than $2 \mathrm{~h}$ ) with functional limitation. There were no associated sicca symptoms. The investigation showed negative viral serology, normal uric acid, ANA 1/160 fine speckled nuclear pattern, normal complement and negative rheumatoid factor, anti-CCP, anti-dsDNA, anti-Sm, anti-RNP, anti-Iupus coagulant, anticardiolopin. Anti-Ro was positive. In imaging, it was observed unaltered hand and wrist radiography. The T2 resonance of the hands/wrists showed synovitis of the wrists and bilateral extensor and flexor tendon tenosynovitis (Figure 1). The main diagnostic hypothesis was polyarthritis secondary to $\mathrm{CPI}$, since there was biological plausibility due to the mechanism of action, marked temporal relationship with drug infusion start, and similar reports in the literature. Sjögren's syndrome was considered due to ANA and anti-Ro positives, but there was no other evidence for the disease. Viral causes, sarcoidosis and microcrystalline arthritis were also excluded. It was prescribed IM betamethasone with significant clinical improvement (VAS for pain from 10 to 2), the pembrolizumab dose was delayed by 2 weeks and prescribed prednisone $10 \mathrm{mg} /$ day, with progressive dose reduction and persistent symptom control.

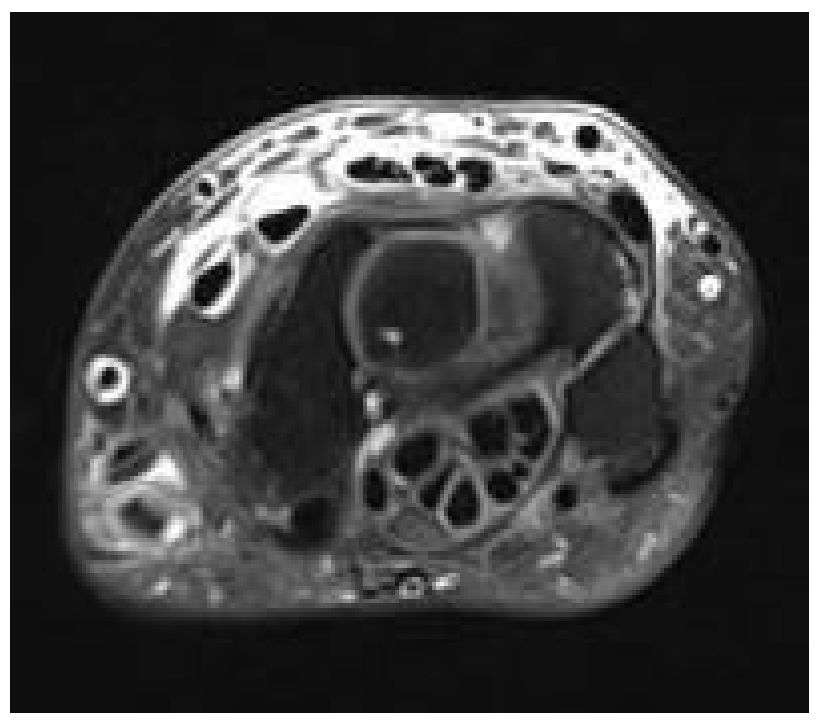

Figure 1. T2 resonance of the hands/wrists show synovitis of the wrists and bilateral extensor and flexor tendon tenosynovitis. 


\section{CONCLUSION}

Checkpoint inhibitors, by promoting activation of the immune system against tumor cells, can also promote activation against selfantigens and immune-mediated adverse events, challenging rheumatologists in diagnosis of rheumatic manifestations. Polyarthritis and its appropriate management are important for improving quality of life of patients with concomitant advanced cancer treated with CPI. Shared decision with the oncologist in follow-up is mandatory.

\section{KEYWORDS}

Immunotherapy, Checkpoint inhibitors, Pembrolizumab, Arthritis. 